\title{
Solid Dispersion Incorporated Microcapsules: Predictive Tools for Improve the Half Life and Dissolution Rate of Pioglitazone Hydrochloride
}

\author{
Singh Chhater ${ }^{1, *}$, Kumar Praveen ${ }^{2}$ \\ ${ }^{1}$ Shri Venkateshwara University Rajabpur, Gajraula/SVU, (U.P.) India \\ ${ }^{2}$ S.D. College of Pharmacy \& Vocational Studies, Muzaffarnagar, (U.P.) India \\ *Corresponding author: pharma_pharm@yahoo.com
}

Received May 05, 2013; Revised May 26, 2013; Accepted May 29, 2013

\begin{abstract}
The present study was aimed to formulate the solid dispersion incorporated microcapsule to improve the dissolution rate and half life of pioglitazone hydrochloride. The solvent evaporation method was used to formulate the solid dispersion resulted increased dissolution rate, bioavailability and stability. Finally increase the half life of the drug by employ the orifice ionic gelation method to formulate solid dispersion incorporated muco-adhesive microcapsule. The solubility of pioglitazone hydrochloride was increase by the preparation of its solid dispersion with polyvinyl pyrrolidone K30 using solvent evaporation methods. The microcapsules of pioglitazone hydrochloride were prepared by (orifice ionic gelation method) employing sodium alginate as a cell forming polymer and using a different bio-adhesive polymers as carbopol, HPMC and sodium CMC in a various ratios of 1:1, 3:1, 6:1 \& 9:1, by orifice ion gelation method. FT-IR spectra revealed no chemical incompatibility between drug and polymers. Drug-polymer interactions were investigated using differential scanning calorimetry (DSC), Powder XRay Diffraction (PXRD). Scanning electron microscope photographs of samples revealed that all prepared microcapsules were almost spherical in shape and have a slightly smooth surface.
\end{abstract}

Keywords: solid dispersion, muco-adhesive microcapsules, orifice gelation method, solvent evaporation method, biological half life

\section{Introduction}

Pioglitazone is a Thiazolidinedione antidiabetic agent that depends on the presence of insulin for its mechanism of action [1,2]. Pioglitazone is a potent and highly selective agonist for peroxisome proliferator-activated receptor-gamma (PPARg) [3]. Pioglitazone decreases insulin resistance in the periphery and in the liver resulting in increased insulin-dependent glucose disposal and decreased hepatic glucose output. Pioglitazone $\mathrm{HCl}$ is a poor water soluble drug and has a short half life (3-5 hour). The formulation of poorly water-soluble drugs has always been a challenging problem faced by pharmaceutical scientists and it is expected to increase because approximately $40 \%$ or more of the new chemical entities being generated through drug discovery programs are poorly water-soluble. It is necessary to improve the solubility and bioavailability and half life.

In the present study, an attempt was made to develop solid dispersion incorporated to increase the dissolution rate and half-life of drug by using solvent evaporation method for sold dispersion and orifice ionic gelation method for solid dispersion incorporated microcapsules. Microencapsulation has been accepted as a process to achieve controlled release and drug targeting. The choice of the methods for the preparation of microcapsules depends on many factors such as the drug solubility and its short half life 3-5 hour [2] and is eliminated rapidly. Mucoadhesion has been a topic of interest in the design of drug delivery system to prolong the residence time of the dosage form at the site of application or the absorption and to facilitate intimate contact dosage form with the underlying absorption surface to improve and enhance the bioavailability of drugs $[4,5,6]$.

\section{Materials and Methods}

Pioglitazone $\mathrm{HCl}$ sample from Ontop Pharmaceuticals LTD (Bangalore, India), Sodium carboxymethylcellulose (sodium CMC), Methyl cellulose (Mc), Hydroxypropylmethylcellulose (HPMC), Polyvinyl pyrrolidone K30 (PVP K30) and Polyethylene glycol 6000 (PEG 6000) was purchase in the market; all the chemicals were A.R. Grade.

\subsection{Estimation of Drug}

\subsubsection{Linear Regression Equation Method}

Accurately weighed about 100mg of pioglitazone hydrochloride was dissolved in $100 \mathrm{ml}$ Phosphate buffer (pH 7.4) to obtain $1000 \mu \mathrm{g} / \mathrm{ml}$ concentration of drug (stock A). Stock A (10ml) was diluted up to $1000 \mathrm{ml}$ with solvent system to obtain $10 \mu \mathrm{g} / \mathrm{ml}$ concentration (Stock B). Aliquots of Stock B were diluted to obtain concentrations 
of $1,2,3,4,5$ to $10 \mu \mathrm{g} / \mathrm{ml}$ of pioglitazone hydrochloride. [7,8] All dilutions were scanned from 400 to $200 \mathrm{~nm}$ against solvent system as blank (Figure 1A) and their absorbance were observed at 269nm (Figure 1B). The LRE was developed as $\mathrm{Y}=0.0202 \mathrm{X}+0.0013$, where $\mathrm{ABC}=$ absorbance and $\mathrm{C}=$ concentration of dilutions in $\mu \mathrm{g} / \mathrm{ml}$, with the correlation coefficient $\mathrm{r}^{2}=0.9998$.

\subsubsection{Standard Absorptivity Method}

Five dilutions were prepared in triplicate and the absorbance was observed at $269 \mathrm{~nm}$. The standard absorptivity e $(1 \%, 1 \mathrm{~cm})$ was calculated from the above observations (Table 1).
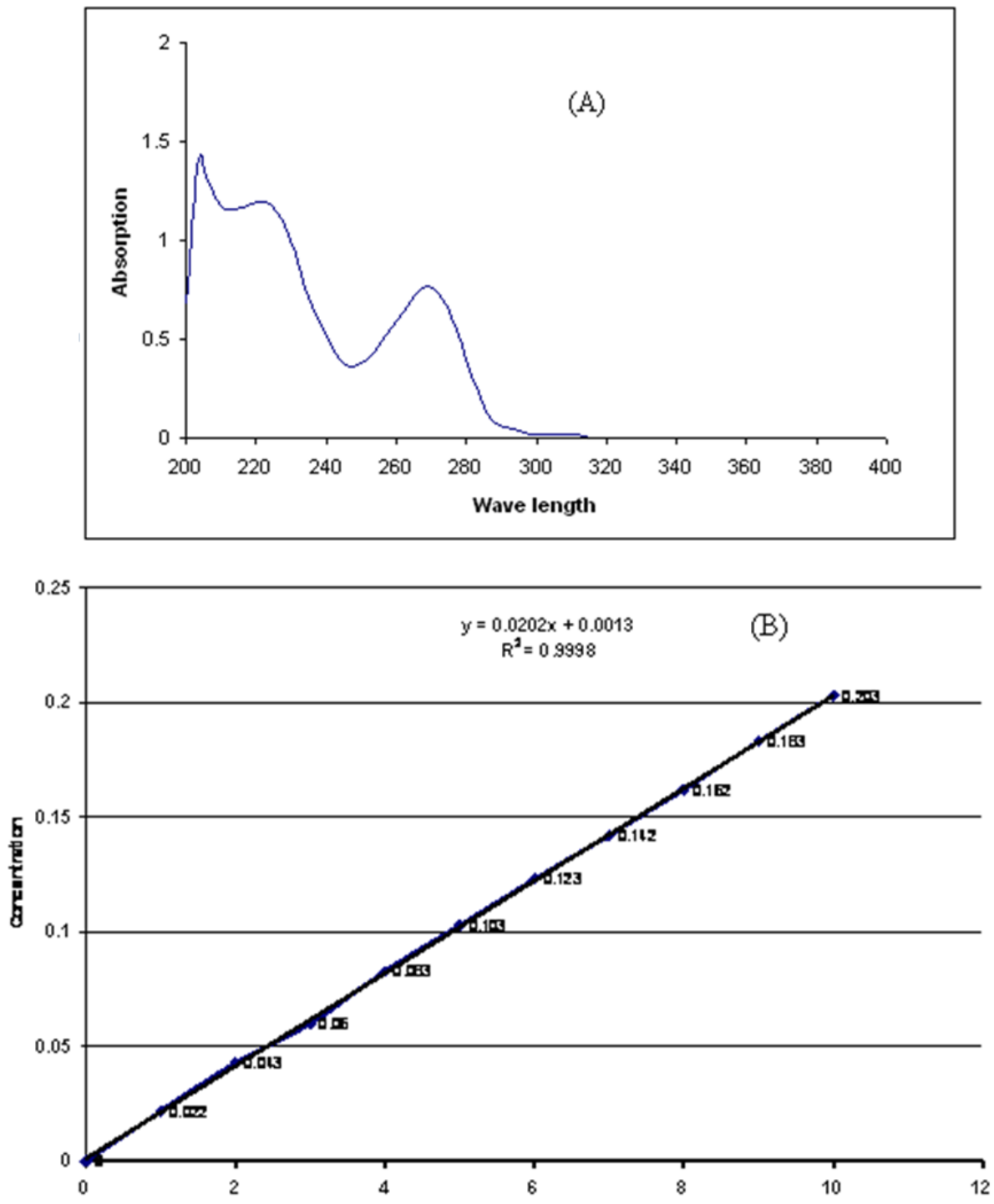

Figure 1. (A) Spectra of pioglitazone $\mathrm{HCl}$ in phosphate buffer (pH 7.4), (B) Calibration graph of pioglitazone $\mathrm{HCl}$

Table 1. Data of absorption and standard absorptivity at different concentration

\begin{tabular}{|c|c|c|c|c|c|c|}
\hline \multirow{2}{*}{$\begin{array}{l}\text { Concentration } \\
(\mathrm{mcg} / \mathrm{ml})\end{array}$} & \multicolumn{3}{|c|}{ Absorption } & \multicolumn{3}{|c|}{ Standard Absorptivity $(\mathrm{e}=\mathrm{A} / \mathrm{BC})$} \\
\hline & $\mathrm{I}$ & II & III & $\mathrm{I}$ & II & III \\
\hline 1 & 0.022 & 0.021 & 0.023 & 220.0 & 210.0 & 230.0 \\
\hline 2 & 0.043 & 0.045 & 0.046 & 215.0 & 225.0 & 230.0 \\
\hline 3 & 0.06 & 0.064 & 0.067 & 200.0 & 213.3 & 223 \\
\hline 4 & 0.083 & 0.082 & 0.085 & 207.5 & 205.0 & 212.5 \\
\hline 5 & 0.103 & 0.107 & 0.105 & 206.0 & 214.0 & 210.0 \\
\hline 6 & 0.123 & 0.127 & 0.120 & 205.0 & 211.6 & 200.0 \\
\hline 7 & 0.142 & 0.146 & 0.139 & 202.8 & 208.5 & 198.5 \\
\hline 8 & 0.162 & 0.164 & 0.167 & 202.5 & 205.0 & 208.7 \\
\hline 9 & 0.183 & 0.185 & 0.178 & 203.3 & 205.5 & 197.7 \\
\hline 10 & 0.203 & 0.205 & 0.208 & 203.0 & 205.0 & 208.0 \\
\hline
\end{tabular}

\subsection{Phase Solubility Study}

Solubility studies were performed according to the Higuchi [9] and Connors method. An excess amount of Pioglitazone hydrochloride was placed in to $50 \mathrm{ml}$ flask containing different concentration of polyvinyl pyrrolidone
K30 and poly ethylene glycol 6000 separately in $25 \mathrm{ml}$ distilled water. All flasks were closed with stopper and covered with cellophane membrane to avoid solvent lose. The flasks were kept in the incubator shaker for $72 \mathrm{~h}$. After $72 \mathrm{~h}$ the content of each flask was then filtered through Whatman filter paper; the filtrate was diluted and assayed 
spectrophotometrically (Shimadzu 1700UV spectrophotometer) measurements were performed in triplicate (Figure 2). for Pioglitazone $\mathrm{HCl}$ content at 269nm. All solubility

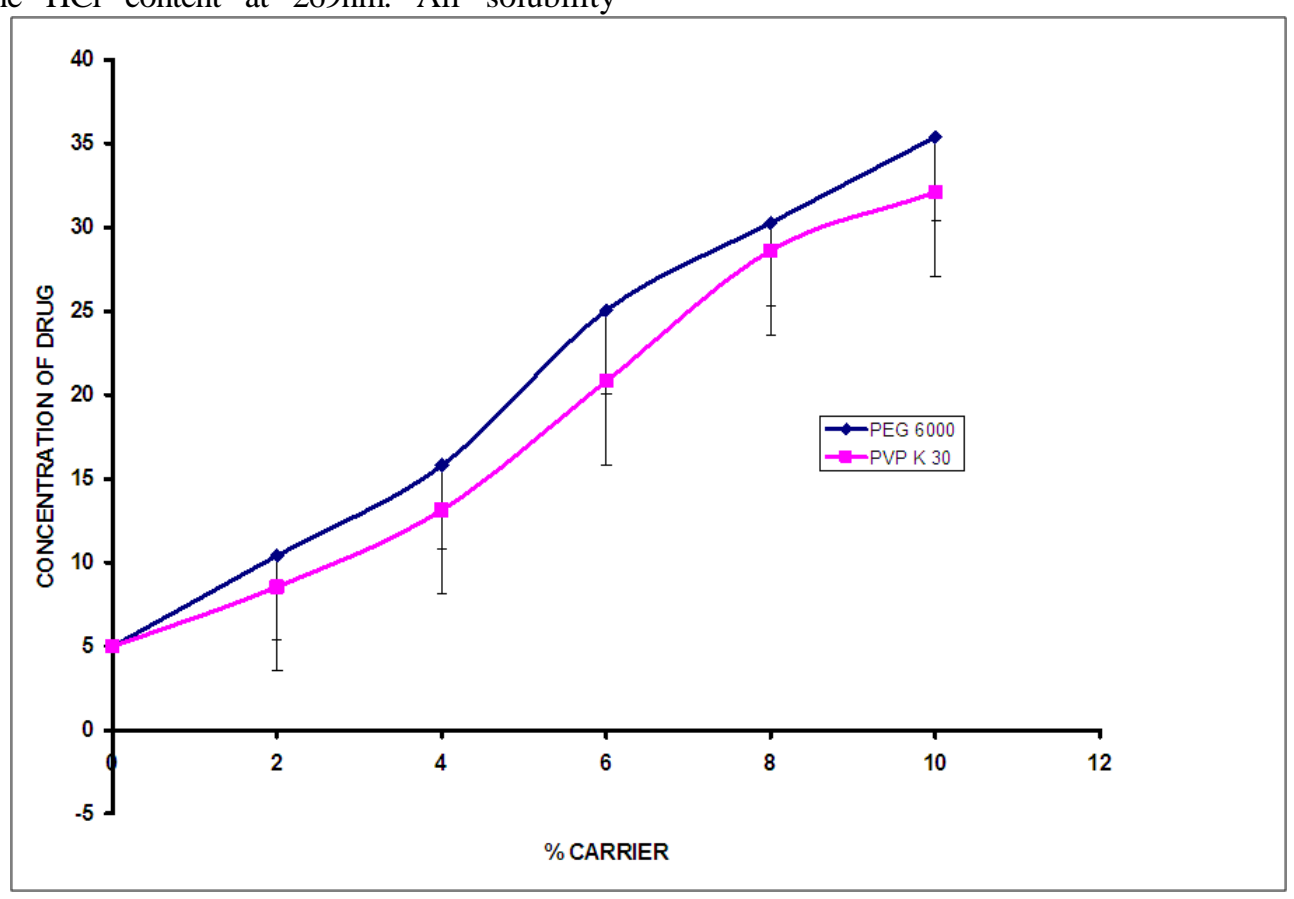

Figure 2. Phase solubility diagram of Pioglitazone hydrochloride in different carrier

\subsection{Preparation of Method}

\subsubsection{Preparation of Physical Mixture and Solid Dispersions}

Physical mixtures were prepared by mixing the appropriate amount of Pioglitazone $\mathrm{HCl}$ and Polyvinyl pyrrolidone K30 (PVP K30) and Polyethylene glycol 6000 (PEG 6000) in pestle and mortar separately and passed through sieve \# 60. Solid dispersions were prepared by solvent evaporation method. The carrier (PVP K30 and PEG 6000) and adding amounts of Pioglitazone $\mathrm{HCl}$ corresponding to ratio $1: 1,2: 1,3: 1$ and 5:1 was accurately weighed and mixed properly. This physical mixture was solubilized in a common solvent that is in ethanol $(25 \mathrm{ml})$. The solvent was allowed to evaporate in hot air oven at $45^{\circ} \mathrm{C} \pm 10^{\circ} \mathrm{C}$. The process of evaporation was opted until the constant weight was obtained. This formulation was kept in dessicator for $24 \mathrm{~h}$ under vacuum. Then, solid dispersion formulation was pulverized using a porcelain mortar and pestle. The pulverized powder was classified using the sieves (size 60\# and 120\# mesh) and the particle size fraction of 100-250mm was used for the study (Table 2).

Table 2. Composition of solid dispersions (SD) and physical mixtures (PM) of Pioglitazone Hydrochloride

\begin{tabular}{|c|c|c|c|c|c|c|c|}
\hline S. No. & Name of Carrier & $\begin{array}{l}\text { Product } \\
\text { Name }\end{array}$ & $\begin{array}{l}\text { Drug } \\
\text { (mg) }\end{array}$ & Carrier (mg) & $\begin{array}{c}\text { Drug carrier } \\
\text { Ratio }\end{array}$ & $\begin{array}{l}\text { Description of } \\
\text { product }\end{array}$ & Preparation of method \\
\hline 1. & Polyvinyl pyrrolidone K30 & A11 & 1500 & 1500 & $1: 1$ & Solid Dispersion & Solvent Evaporation \\
\hline 2. & Polyvinyl pyrrolidone K30 & A12 & 1000 & 2000 & $1: 2$ & Solid Dispersion & Solvent Evaporation \\
\hline 3. & Polyvinyl pyrrolidone K30 & A13 & 750 & 2250 & $1: 3$ & Solid Dispersion & Solvent Evaporation \\
\hline 4. & Polyvinyl pyrrolidone K30 & A15 & 500 & 2500 & $1: 5$ & Solid Dispersion & Solvent Evaporation \\
\hline 5. & Polyvinyl pyrrolidone K30 & PMA15 & 500 & 1500 & $1: 3$ & Physical Mixture & ---------------- \\
\hline 6. & Polyethylene Glycol 6000 & B11 & 1500 & 1500 & $1: 1$ & Solid Dispersion & Solvent Evaporation \\
\hline 7. & Polyethylene Glycol 6000 & B12 & 1000 & 2000 & $1: 2$ & Solid Dispersion & Solvent Evaporation \\
\hline 8. & Polyethylene Glycol 6000 & B13 & 750 & 2250 & $1: 3$ & Solid Dispersion & Solvent Evaporation \\
\hline 9. & Polyethylene Glycol 6000 & B15 & 500 & 2500 & $1: 5$ & Solid Dispersion & Solvent Evaporation \\
\hline 10. & Polyethylene Glycol 6000 & PMB13 & 500 & 1500 & $1: 3$ & Physical Mixture & ........ \\
\hline
\end{tabular}

2.3.2. Preparation of Solid Dispersion Incorporated Muco-Adhesive Microcapsules

Microcapsules are prepared by orifice- ionic gelation method $[10,11]$ by employing the Sodium alginate as a cell forming polymer and Sodium CMC, HPMC and Carbopol as muco-adhesive [12] polymers are dissolved in purified water in a corresponding ratio 1:1, 1:3 and 6:1 separately to form a homogenous polymer solution. Core material (solid dispersion of pioglitazone hydrochloride) equivalent to drug (1gm) is added to polymer solution and mixed thoroughly with a stirrer to form a viscous dispersion. The resulting dispersion is then added manually drop wise into $\mathrm{CaCl}_{2}(10 \% \mathrm{w} / \mathrm{v})$ solution through a syringe with a needle of size no. 18. The added drop lets are related in the $\mathrm{CaCl}_{2}$ solution for 15 min to complete the curing reactions and to produce spherical rigid microcapsule. The microcapsules are collected by decantation, and the product thus separated, washed repeatedly with water and dried at $45^{\circ} \mathrm{C}$ for $12 \mathrm{hrs}$. (Table 3). 
Table 3. Composition of different muco-adhesive microcapsules

\begin{tabular}{|c|c|c|c|c|}
\hline $\begin{array}{c}\text { Formulation } \\
\text { code }\end{array}$ & $\begin{array}{c}\text { Composition } \\
\text { and ratio }\end{array}$ & $\begin{array}{c}\text { SD (A13) } \\
\text { equivalent to mg of drug }\end{array}$ & $\begin{array}{c}\text { Cell forming polymer } \\
\text { (mg) }\end{array}$ & $\begin{array}{c}\text { Mucoadhesive Polymer } \\
\text { (mg) }\end{array}$ \\
\hline MC1 & SA: SCMC (1:1) & 1000 & 500 & 500 \\
\hline MC2 & SA: HPMC (1:1) & 1000 & 500 & 500 \\
\hline MC3 & SA: Carbopol (1:1) & 1000 & 500 & 500 \\
\hline MC4 & SA: SCMC (3:1) & 1000 & 750 & 250 \\
\hline MC5 & SA: HPMC (3:1) & 1000 & 750 & 250 \\
\hline MC6 & SA: Carbopol (3:1) & 1000 & 750 & 250 \\
\hline MC7 & SA : SCMC (6:1) & 1000 & 857.14 & 142.86 \\
\hline MC8 & SA : HPMC (6:1) & 1000 & 857.14 & 142.86 \\
\hline MC9 & SA : Carbopol (6:1) & 1000 & 857.14 & 142.86 \\
\hline
\end{tabular}

Note: Sodium alginate $=$ SA, Sodium CMC $=$ SCMC, SD $=$ Solid dispersion

\section{Characterization of Solid Dispersion}

\subsection{Physical Characterization}

The surface and internal structure of solid dispersion was observed through the Scanning Electron microscopy (SEM), by using the scanning electron microscope (SEMLEICA S430, London, UK). (Figure 6E, F).

\subsection{Practical Yield}

The percentage yield of Pioglitazone in solid dispersion was determined by using the formula:

$$
\% \text { Yield }=\frac{\text { Weight of Microcapsules }}{\text { Theortical Weight of drug and polymer }} \times 100
$$

\subsection{Percentage Drug Content}

The dispersion system equivalent to $25 \mathrm{mg}$ of Pioglitazone hydrochloride was taken in $25 \mathrm{ml}$ volumetric

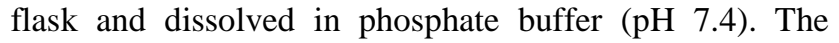
volume was made up to the mark with phosphate buffer (pH 7.4) and filtered. One ml of filtrate was further diluted to $10 \mathrm{ml}$ with phosphate buffer ( $\mathrm{pH} \mathrm{7.4)}$ and absorbance was recorded at $269 \mathrm{~nm}$. The amount of drug in each dispersion system was determined spectrophotometrically (Table 4).

\section{$\%$ Drug content}

Actual drug content of microcapsules

$=\overline{\text { Theoretical Weight of drug and microcapsules }}$
Table 4. Drug Content of solid dispersion system

\begin{tabular}{|c|c|c|c|}
\hline S. No. & Product Name & \% yield & \% Drug content \\
\hline 1 & A11 & 84.91 & 97.54 \\
\hline 2 & A12 & 87.55 & 92.22 \\
\hline 3 & A13 & 82.98 & 95.14 \\
\hline 4 & A15 & 88.67 & 89.07 \\
\hline 5 & PMB13 & 87.90 & 97.54 \\
\hline 6 & B11 & 81.37 & 98.02 \\
\hline 7 & B12 & 86.56 & 91.17 \\
\hline 8 & B13 & 89.39 & 86.52 \\
\hline 9 & B15 & 82.99 & 83.37 \\
\hline 10 & PMB13 & 85.81 & 96.02 \\
\hline
\end{tabular}

\subsection{In Vitro Drug Release}

\subsubsection{Release in $\mathbf{p H}$ 7.4-Phosphate Buffer}

In vitro release rate of Pioglitazone $\mathrm{HCl}$ alone and Pioglitazone $\mathrm{HCl}$ from solid dispersion of different samples was determined using single station USP dissolution test apparatus. The dissolution medium consisted of phosphate buffer (pH 7.4) was used. Samples of drug, solid dispersion equivalent with $100 \mathrm{mg}$ of drug were spread onto the surface of $900 \mathrm{ml}$ of preheated dissolution medium at $37^{\circ} \mathrm{C}$. Aliquots of $2 \mathrm{ml}$ were withdrawn at regular intervals of time i.e. (5, 10, 15, 20, up to 120min) and the same is replaced with fresh dissolution medium each time. The samples obtained were filtered through Whatman filter paper no. 1. The filtrate was diluted up to $6 \mathrm{ml}$ with phosphate buffer ( $\mathrm{pH} 7.4$ ). Then the absorbance was measured at $269 \mathrm{~nm}$.

Table 5. Drug release kinetics data of different formulations

\begin{tabular}{|c|c|c|c|c|c|c|}
\hline \multirow{2}{*}{ Formulation code } & \multicolumn{6}{|c|}{ Regression Coefficient (r) value } \\
\hline & Zero order & First order & Weibull & Korsmeyer-peppas & Hill equation & Michaelis-menten \\
\hline A11 & 0.2239 & 0.1183 & 0.9873 & 0.9859 & 0.9892 & 1.0000 \\
\hline A12 & 0.3320 & 0.1223 & 0.9901 & 0.9897 & 0.9927 & 1.0000 \\
\hline A13 & 0.3499 & 0.1223 & 0.9907 & 0.9876 & 0.9945 & 1.0000 \\
\hline A15 & 0.3023 & 0.1203 & 0.9888 & 0.9864 & 0.9920 & 1.0000 \\
\hline PMA13 & 0.6156 & 0.1309 & 0.9922 & 0.9903 & 0.9943 & 1.0000 \\
\hline B11 & 0.2520 & 0.1192 & 0.9880 & 0.9863 & 0.9903 & 1.0000 \\
\hline B12 & 0.2689 & 0.1194 & 0.9877 & 0.9861 & 0.9900 & 1.0000 \\
\hline B13 & 0.2943 & 0.1202 & 0.9892 & 0.9865 & 0.9927 & 1.0000 \\
\hline B15 & 0.2686 & 0.1194 & 0.9878 & 0.9861 & 0.9901 & 1.0000 \\
\hline PMB13 & 0.4870 & 0.1287 & 0.9928 & 0.9904 & 0.9955 & 1.0000 \\
\hline MC1 & 0.9516 & 0.7781 & 0.9888 & 0.9600 & 0.9859 & 0.9484 \\
\hline MC2 & 0.9774 & 0.7993 & 0.9937 & 0.8988 & 0.9404 & 0.9289 \\
\hline MC3 & 0.9752 & 0.7383 & 0.9871 & 0.9340 & 0.9522 & 0.9041 \\
\hline MC4 & 0.9650 & 0.7526 & 0.9854 & 0.9645 & 0.9158 & 0.9735 \\
\hline MC5 & 0.9279 & 0.7015 & 0.9899 & 0.9310 & 0.9443 & 0.9406 \\
\hline MC6 & 0.9311 & 0.6859 & 0.9786 & 0.9159 & 0.9571 & 0.9296 \\
\hline MC7 & 0.9611 & 0.7667 & 0.9949 & 0.9732 & 0.9571 & 0.9888 \\
\hline MC8 & 0.9503 & 0.7664 & 0.9940 & 0.9722 & 0.9538 & 0.9903 \\
\hline MC9 & 0.9492 & 0.7562 & 0.9951 & 0.9691 & 0.9578 & 0.9889 \\
\hline
\end{tabular}




\subsubsection{Drug Release Kinetics Studies}

In order to understand the kinetics and mechanism of drug release, the results of the in vitro drug release study were fitted with various kinetic equations like zero order, first order, Weibull model, Korsmeyer -peppas model, Hill equation, Michaelis- Menten model. The kinetic model that best fits the dissolution data was evaluated by comparing the regression coefficient (r) values obtained in various models. (Table 5).

\section{\%CDR Dü VI frmulatian}

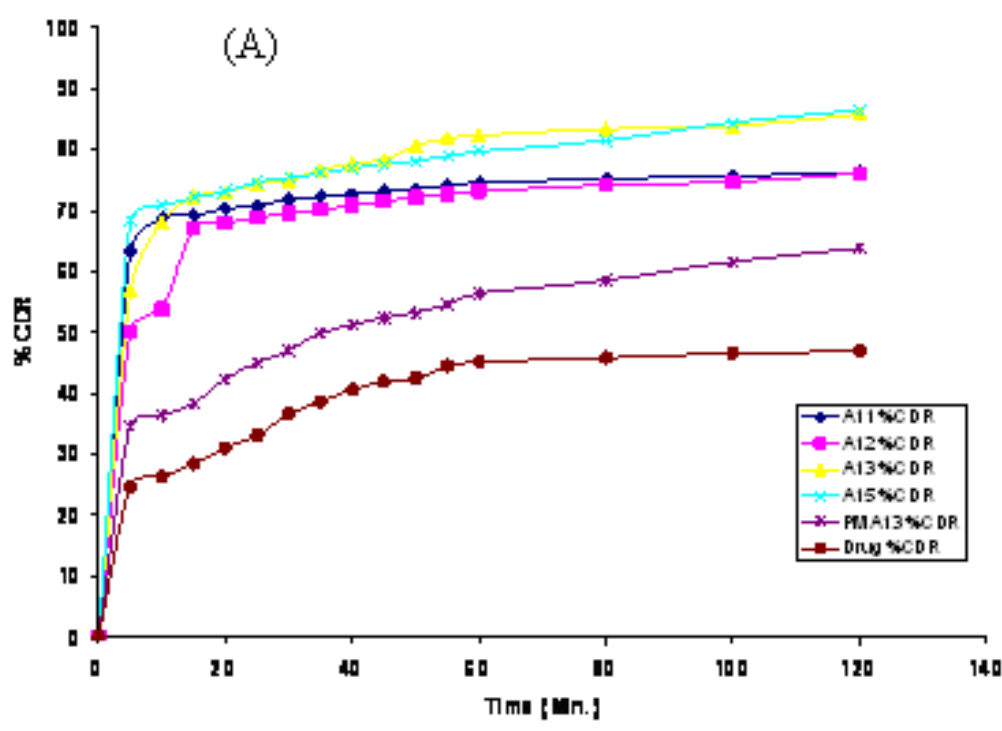

\%egh Dru Vhe Fumbiation
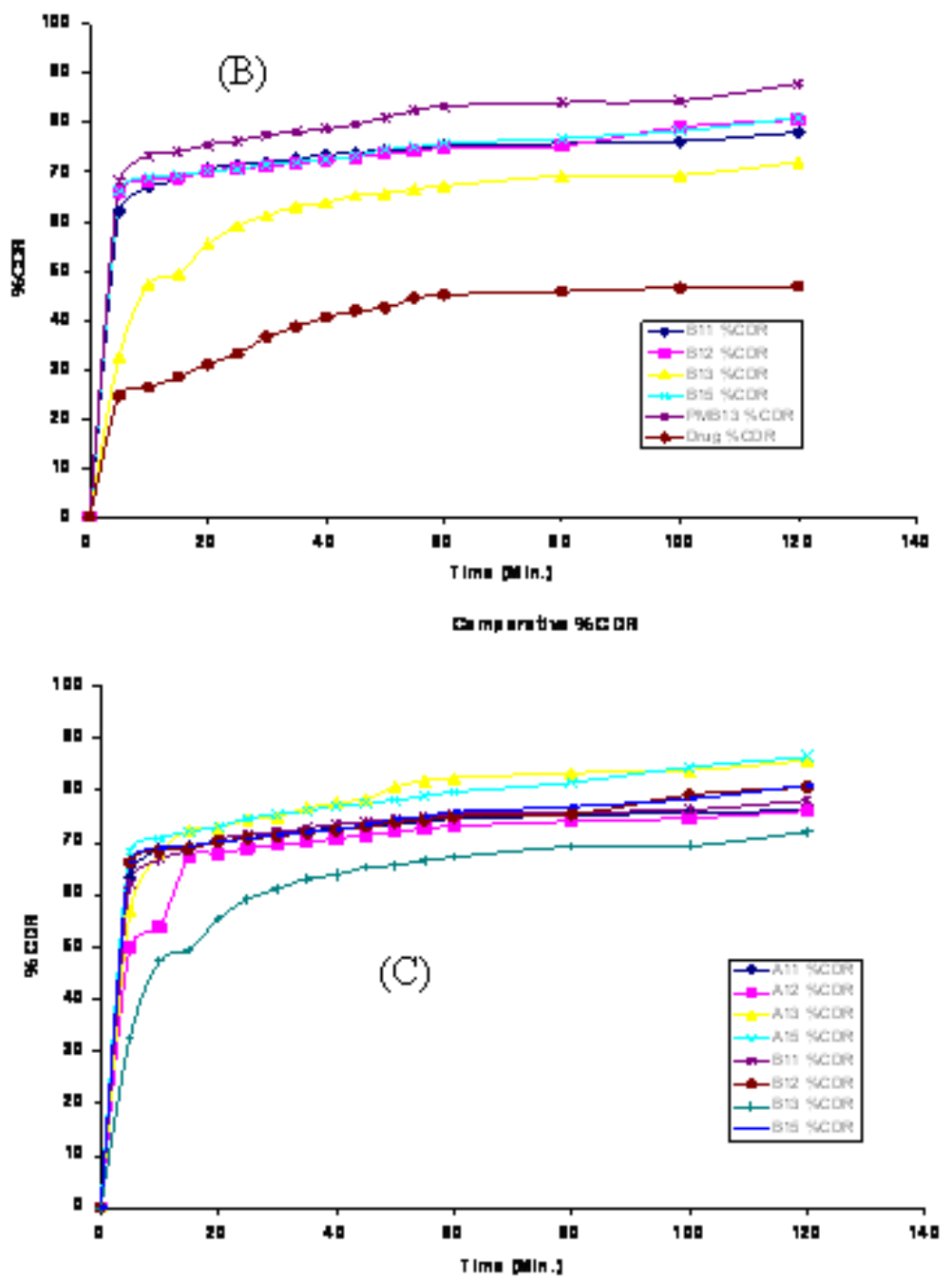

Figure 3. (A) Comparative \% release of pure drug and different formulations containing PVP K30 as carrier, (B). Comparative \% release of pure drug and different formulations containing PEG 6000 as carrier, (C) Comparative \% CDR of different formulations 


\subsection{Analytical Testing of Solid Dispersion}

\subsubsection{Fourier Transforms Infrared Spectroscopy}

FT-IR spectra $\left(500-4000 \mathrm{~cm}^{-1}\right)$ were obtained on a Nicolet Avatar 37-DTGS FT-IR spectrophotometer (Nicolet) with a resolution of $4 \mathrm{~cm}^{-1}$. KBr pellets were prepared by gently mixing $1 \mathrm{mg}$ sample with $200 \mathrm{mg}$ potassium bromide.

\subsubsection{X-Ray Diffraction}

Diffraction patterns were obtained at room temperature on a Philips PW 1710 Diffractrometer (Philips, Holland). Samples were exposed to $\mathrm{Cu} \mathrm{Ka}$ radiation, wavelength $1.54060 \AA$ A through 1 x slits from 5.025 to 59.6852 q with a step size of $0.60^{\circ} 2 \mathrm{q}$ and a count time of 1 sec. per step; the generator was set to $40 \mathrm{kV}$ and $30 \mathrm{~mA}$.

\section{Characterization of Microcapsules}

\subsection{Physical Characterization $[13,14]$}

The surface and inner part of the microspheres was observed through the Scanning Electron microscopy (SEM), (SEM) was performed for surface and inner morphological characterization of microspheres using the scanning electron microscope (SEM- LEICA S430, London, UK). (Figure 6).

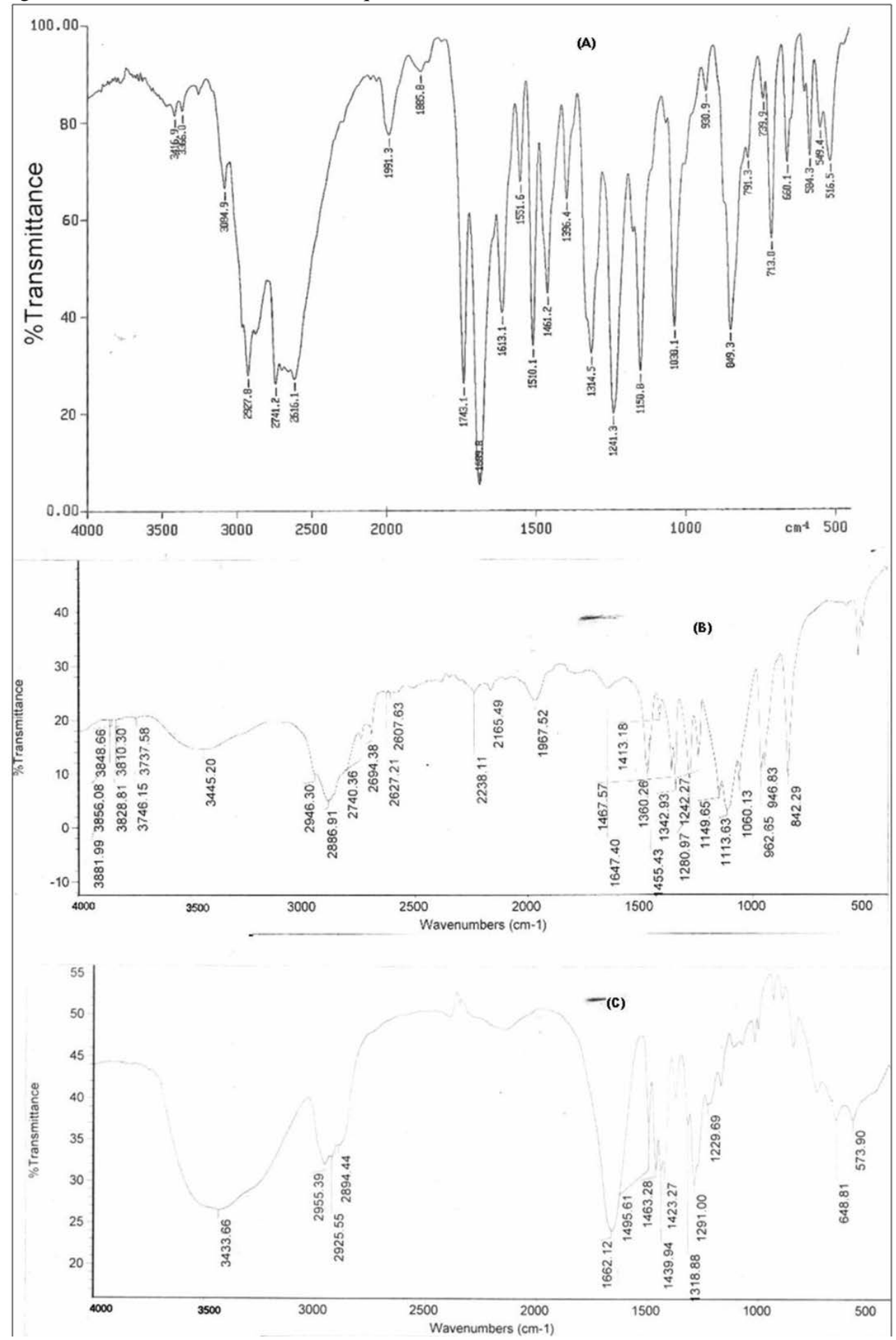




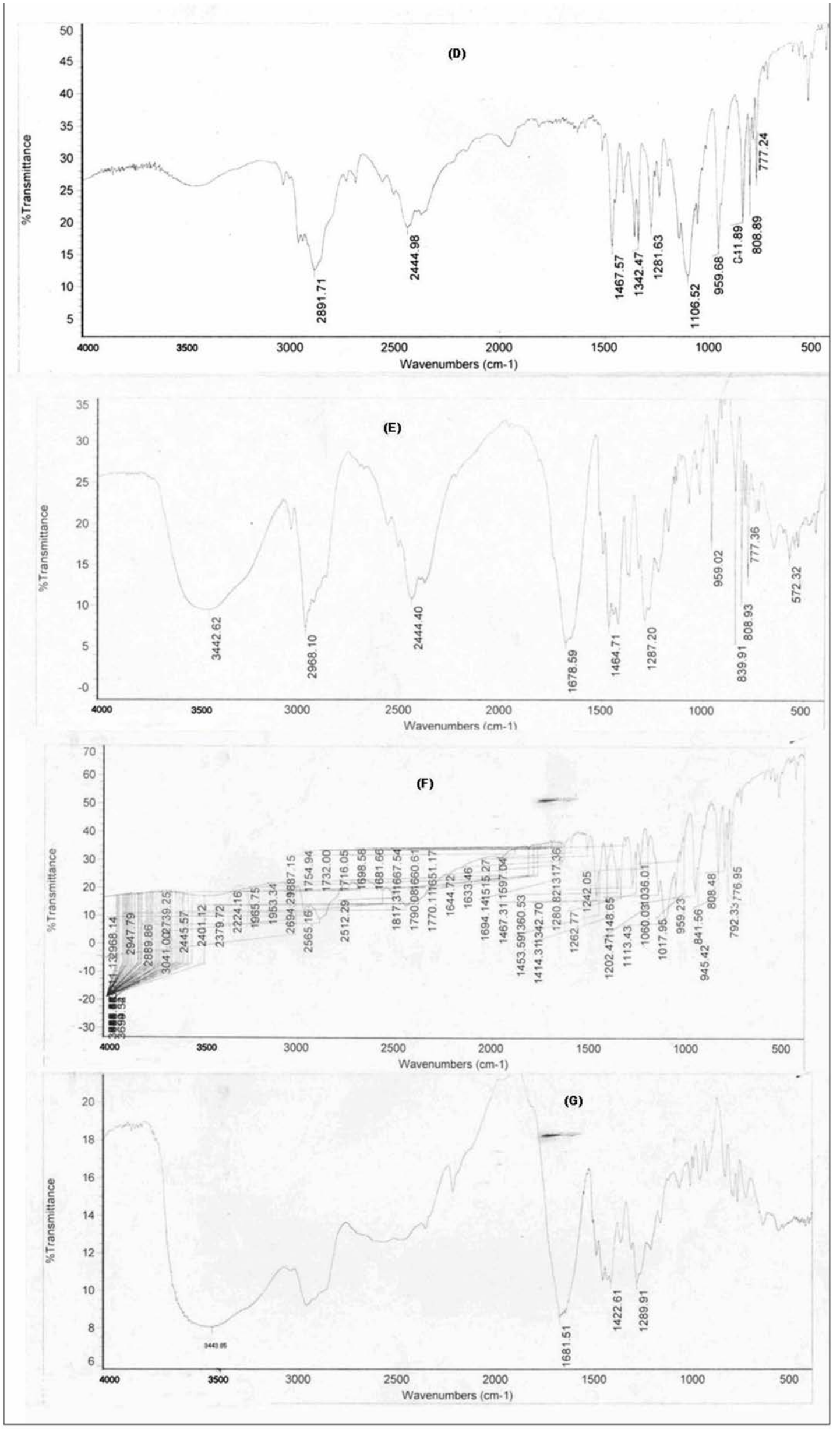

Figure 4. FTIR Spectra of (a). Pioglitazone HCL, (B). PVP K30, (C). PEG 6000, (D). PMA13, (E). PMB13, (F). A13, (G). B13 


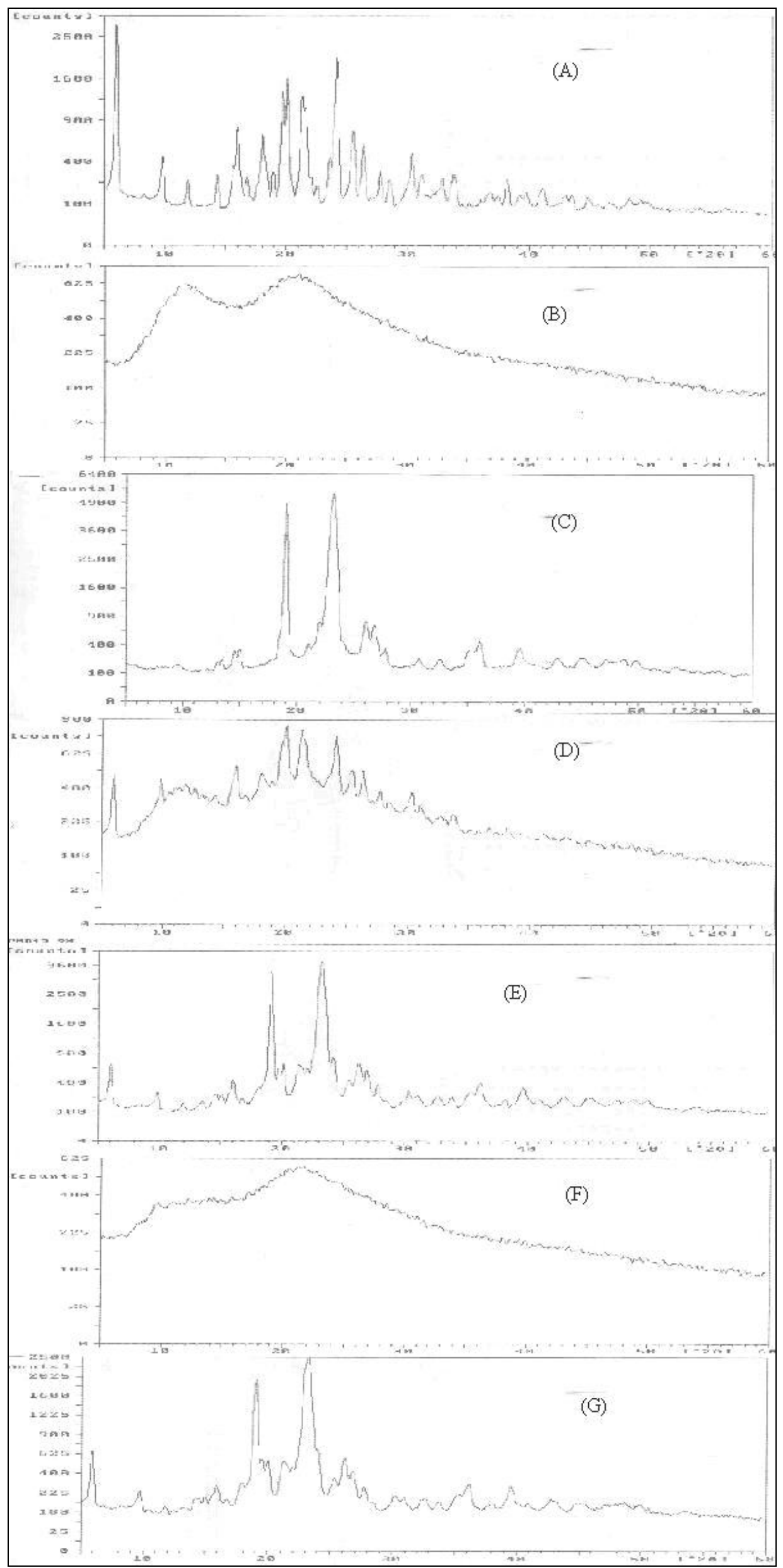

Figure 5. XRD Spectra of (a) Pioglitazone HCL, (B) PVP K30, (C) PEG 6000, (D) PMA13, (E) PMB13, (F) A13, (G) B13 


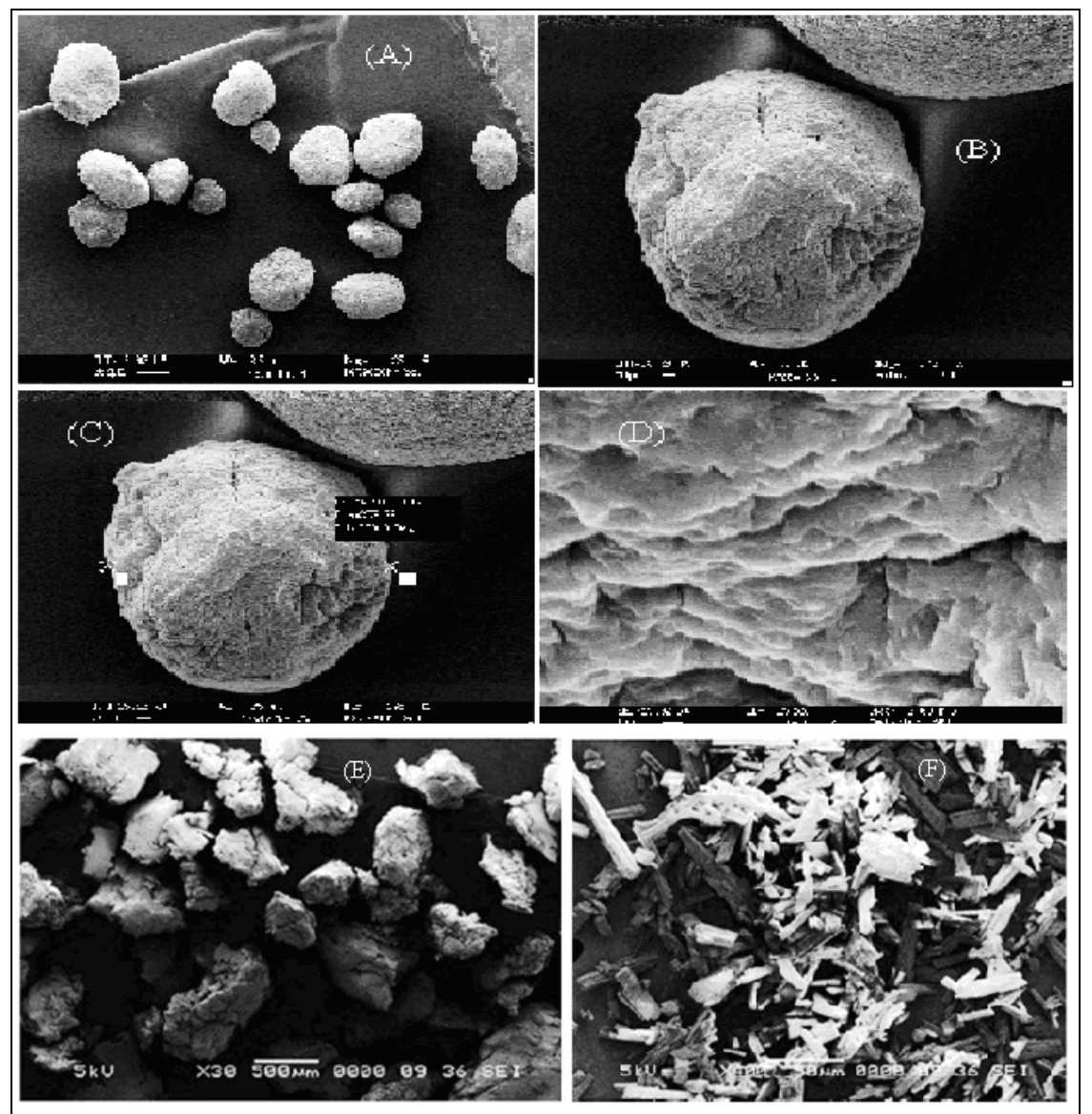

Figure 6. SEM photographs of Pioglitazone (A) microcapsules with size ranging approximately from 300 to $900 \mu \mathrm{m}$, (B) Individual microcapsules (C) microcapsules showing the size $353.33 \mu \mathrm{m}$, (D) Surface topography analysis of microcapsules, (E) Internal structure of solid dispersion of sample B13 and (F) show the amorphous and A13 show the crystalline stage

\subsection{Particle Size Distribution}

Different sizes of microcapsules in a batch were separated by sieving method using a range of standard sieves (\#10, \#22, \#44, \#52 and \# 60). The amount retained on different sieves was weighed. From the obtained data, weight percent retained on different sieves and average size of microcapsules were calculated.

\subsection{Practical Yield:}

The percentage yield of Pioglitazone in the microencapsulated product is determined by using the formula:

$$
\% \text { Yield }=\frac{\text { Weight of Microcapsules }}{\text { Theortical Weight of drug and polymer }} \times 100
$$

Table 6. Characterization of muco-adhesive microcapsules

\begin{tabular}{|c|c|c|c|c|c|}
\hline $\begin{array}{l}\text { Formulation } \\
\text { code }\end{array}$ & $\begin{array}{c}\text { Composition } \\
\text { and ratio }\end{array}$ & $\begin{array}{l}\text { Average size } \\
(\mu \mathrm{m})\end{array}$ & $\begin{array}{c}\% \\
\text { yield } \\
\end{array}$ & Drug content (mg) & $\begin{array}{c}\text { Encapsulation } \\
\% \text { efficiency }\end{array}$ \\
\hline MC1 & SA: SCMC (1:1) & 350 & 80.67 & 185.7 & 74.28 \\
\hline MC2 & SA: HPMC (1:1) & 347 & 83.77 & 195.425 & 78.17 \\
\hline MC3 & SA: Carbopol (1:1) & 389 & 87.11 & 169.775 & 67.91 \\
\hline MC4 & SA: SCMC (3:1) & 410 & 89.12 & 182.8 & 73.12 \\
\hline MC5 & SA: HPMC (3:1) & 387 & 81.27 & 201.925 & 80.77 \\
\hline MC6 & SA: Carbopol (3:1) & 341 & 86.25 & 190.625 & 76.25 \\
\hline MC7 & SA : SCMC (6:1) & 354 & 79.87 & 202.6 & 81.04 \\
\hline MC8 & SA: HPMC (6:1) & 372 & 86.82 & 198.325 & 79.33 \\
\hline MC9 & SA: Carbopol (6:1) & 345 & 89.77 & 197.275 & 78.91 \\
\hline
\end{tabular}

\subsection{Percentage Drug Content}

About 500mg of microcapsule was accurately weighed and transfer in to $1000 \mathrm{ml}$ beaker, which contain $900 \mathrm{ml}$ of 7.4 phosphate buffer at $37^{\circ} \mathrm{C}$. The phosphate solution was steered continuously until all the microcapsules were dissolved. Drug loading was determined by U.V Photometric method at 269nm.

(Microencapsulation efficiency) was calculated by the following formula:
\%Drug content

$=\frac{\text { Actual drug content of microcapsules }}{\text { Theoretical Weight of drug and microcapsules }} \times 100$

\subsection{Encapsulation Efficiency}

The encapsulation efficiency of microcapsules was calculated by using the formula:

\%Encapsulation efficiency

$$
=\frac{\% \text { Drug content }}{\% \text { theoretical drug Content }} \times 100
$$




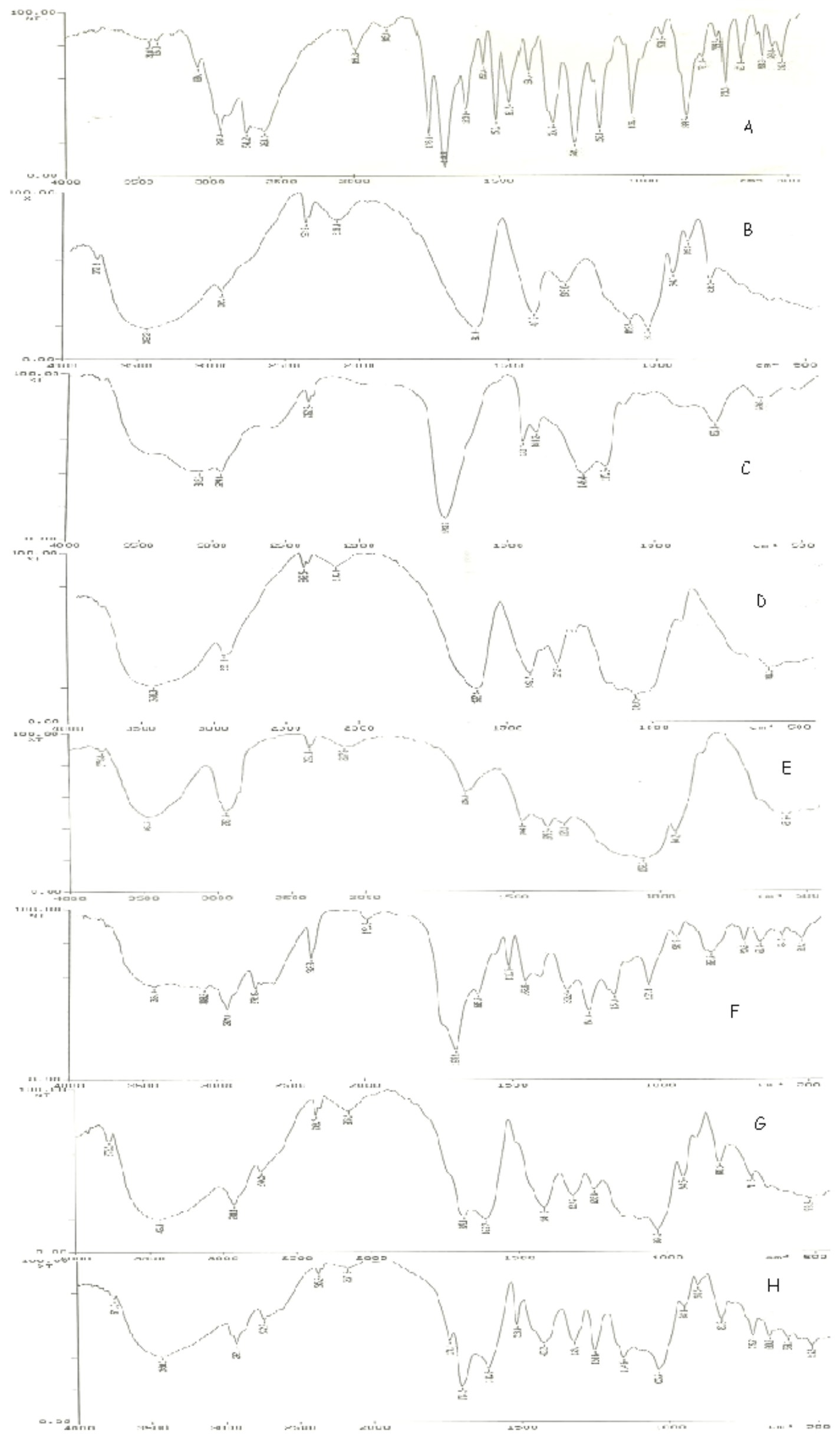

Figure 7. FIR of (A) Pioglitazone HCl, (B) Sodium alginate, (C) Carbopol, (D) Sodium CMC, (E) HPMC, (F) Mixture of A, B and C, (G) Mixture of A, $\mathrm{B}$ and $\mathrm{D},(\mathrm{H})$ Mixture of $\mathrm{A}, \mathrm{B}$ and $\mathrm{E}$ 


\subsection{Fourier Transforms Infrared Spectroscopy}

FT-IR spectra (500-4000 $\left.\mathrm{cm}^{-1}\right)$ were obtained on a Nicolet Avatar 37- DTGS FT-IR spectrophotometer (Nicolet) with a resolution of $4 \mathrm{~cm}^{-1}$. $\mathrm{KBr}$ pellets were prepared by gently mixing $1 \mathrm{mg}$ sample with $200 \mathrm{mg}$ potassium bromide (Figure 7).

\subsection{In-Vitro Drug Release}

\subsubsection{Release in pH 7.4-Phosphate Buffer}

In vitro release rate of Pioglitazone $\mathrm{HCl}$ from microcapsules of different samples was determined using single station USP dissolution test apparatus. The dissolution medium consisted of phosphate buffer ( $\mathrm{pH} 7.4$ ) was used. Samples of drug, microcapsules equivalent with $100 \mathrm{mg}$ of drug was spread onto the surface of $900 \mathrm{ml}$ of preheated dissolution medium at $37^{\circ} \mathrm{C}$. Aliquots of $5 \mathrm{ml}$ were withdrawn at regular intervals of time i.e. (.5, 1, 2, and 3 up to 18 hour) and the same is replaced with fresh dissolution medium each time. The samples obtained were filtered through Whatman filter paper no. 1. The filtrate was diluted up to $6 \mathrm{ml}$ with phosphate buffer ( $\mathrm{pH} 7.4)$. Then the absorbance was measured at 269nm (Figure 8).

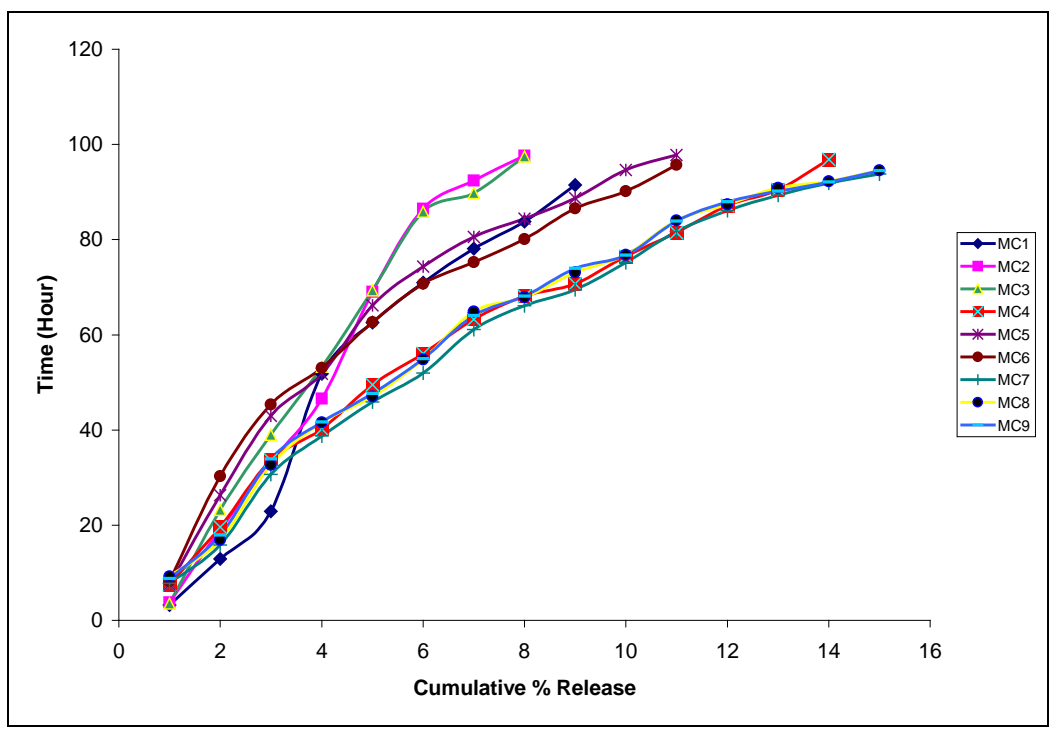

Figure 8. Comparative drug release of various compositions

\subsubsection{Drug Release Kinetics Studies}

In order to understand the kinetics and mechanism of drug release, the results of the in vitro drug release study were fitted with various kinetic equations like zero order, first order, Weibull model, Korsmeyer -peppas model, Hill equation, Michaelis- Menten model. The kinetic model that best fits the dissolution data was evaluated by comparing the regression coefficient (r) values obtained in various models. (Table 5).

Table 7. Microspheres adherence capacity

\begin{tabular}{|c|c|c|c|c|c|}
\hline \multicolumn{5}{|c|}{ Percentage of Microspheres adhering to tissue at pH7.4 } \\
\hline Formulation code & 1 hour & 2 hour & 4 hour & 6 hour \\
\hline MC1 & $72.66 \pm 2.08$ & $70.66 \pm 1.52$ & $54.00 \pm 1.73$ & $32.00 \pm 1.2$ & $22.33 \pm 3.05$ \\
\hline MC2 & $74.66 \pm 1.527$ & $63.57 \pm 1.3$ & $41.66 \pm 3.51$ & $25.33 \pm 1.52$ & $16.00 \pm 3.4$ \\
\hline MC3 & $72.33 \pm 1.52$ & $69.33 \pm 1.15$ & $45.33 \pm 3.05$ & $29.66 \pm 1.527$ & $15.00 \pm 1.7$ \\
\hline MC4 & $72.33 \pm 2.08$ & $61.66 \pm 2.52$ & $49.66 \pm 1.27$ & $11.66 \pm 2.08$ & $11.66 \pm 2.7$ \\
\hline MC5 & $63.66 \pm 3.21$ & $56.66 \pm 0.57$ & $40.00 \pm 1.4$ & $06.70 \pm 2.1$ \\
\hline MC6 & $68.52 \pm 1.3$ & $54.07 \pm 2.08$ & $39.72 \pm 1.3$ & $29.00 \pm 1.52$ & $11.00 \pm 1.4$ \\
\hline MC7 & $67.22 \pm 1.8$ & $41.57 \pm 1.73$ & $35.84 \pm 1.15$ & $10.77 \pm 3.4$ & $02.33 \pm 1.15$ \\
\hline MC8 & $57.97 \pm 1.6$ & $33.50 \pm 1.4$ & $25.36 \pm 1.02$ & $04.37 \pm 1.84$ & ----- \\
\hline
\end{tabular}

\subsection{Mucoadhesive Testing}

The mucoadhesive property of the microcapsules was evaluated by wash-off [15] In vitro method. Freshly excised pieces of intestinal mucosa $(2 \times 2 \mathrm{~cm})$ from sheep were mounted onto glass slides $(3 \times 1$ inch $)$ with cyanoacrylate glue. Two glass slides were connected with a suitable support. About 50 microcapsules were spread onto each wet rinsed tissue specimen, and immediately the slides were hung on to the arm of USP tablet disintegrating test apparatus. The tissue specimen was given a slow, regular up-and-down movement in the test fluid at $37^{\circ} \mathrm{C}$ contained in a $1 \mathrm{~L}$ vessel. After definite time interval, the apparatus was stopped and the number of microcapsules still adhering to the tissue was counted. Phosphate buffer (pH 7.4) was used as test fluid. (Table 7).

\section{Results and Discussion}

\subsection{Solubility Studies of Pioglitazone}

The Solubility of Pioglitazone $\mathrm{HCl}$ in distilled water at $27^{\circ} \mathrm{C}$ was found to be $5.32 \mu \mathrm{g} / \mathrm{mL}$. The influence of polyvinyl pyrrolidone K30 and polyethylene glycol 6000 
upon the solubility of Pioglitazone $\mathrm{HCl}$ is presented in Figure 3 and shows the solubility of Pioglitazone $\mathrm{HCl}$ increases with increase the concentration of both carriers. PVP K30 has the better solubility enhancing capacity than PEG 6000. (Figure 2).

\subsection{Physical Characterization}

The solid dispersion of pioglitazone prepared by solvent evaporation method were found to be discrete and free flowing. The final formulation solid dispersion incorporated microcapsules of pioglitazone prepared by the orifice-ionic gelatin method were found to be discrete, spherical, free flowing, and the monolithic matrix type. The SEM photographs indicated that internal structure of solid dispersion of sample B13 show the amorphous and A13 show the crystalline stage. (Figure $6 \mathrm{E}, \mathrm{F}$ ) and the solid dispersion incorporated microcapsules were uniform in size, with size range of $300 \mu \mathrm{m}$. The SEM photographs indicated that microcapsules were spherical and completely covered the coat polymer (Figure 6-A, B, C, and D).

\subsection{Practical yield of Solid Dispersion}

The percentage practical yield was found to be in the range of 81.37 to $89.39 \%$. The maximum percentage practical yield was found to be $89.39 \%$ for B13. (Table 4).

\subsection{Practical Yield of Solid Dispersion Incorporated Microcapsules}

The percentage practical yield was found to be in the range of 79.87 to $89.77 \%$. The maximum percentage practical yield was found to be $89.77 \%$ for MC-9. (Table 6).

\subsection{Percentage Drug Content of Solid Dispersion}

The actual drug content of all formulations are given in Table the percentage drug content ranges from $83.37 \%$ to $98.02 \%$ for formulation B15 to B11. The maximum percentage drug content was found to be $98.02 \%$ IN B11. (Table 4).

\subsection{Percentage Drug Content and Encapsulation Efficiency of Solid Dispersion Incorporated Microcapsules}

The actual drug content and encapsulation efficiency of all nine formulations are given in Table 6. The encapsulation efficiency ranges from 66.91 to $81.04 \%$ for formulation MC1 to MC9. The maximum encapsulation efficiency was found to be $81.04 \%$ in MC7.

\subsection{Particle Size Distribution of Solid Dispersion Incorporated Microcapsules}

The average size of microcapsules in various batches was found to be $350 \mu \mathrm{m}, 347 \mu \mathrm{m}$, and $389 \mu \mathrm{m}, 410 \mu \mathrm{m}$ $387 \mu \mathrm{m}, 341 \mu \mathrm{m}, 354 \mu \mathrm{m}, 372 \mu \mathrm{m}$ and $345 \mu \mathrm{m}$ for $\mathrm{MC} 1$, MC2, MC3, MC4, MC5, MC6, MC7, MC8 and MC9 respectively.(Table 6).

\subsection{In-Vitro Drug Release}

\subsubsection{In-Vitro Drug Release Study of Solid Dispersion}

Pioglitazone $\mathrm{HCl}$ release from the solid dispersion and alone was studied in phosphate buffer $(\mathrm{pH}$ 7.4) up to 2 hours. The average percentage release of the pure Pioglitazone HCL was found to be $46 \%$ in 2 hours. (Figure 3A, and Figure 3B) In the solid dispersion formulation using polyvinyl pyrrolidone K30 and polyethylene glycol 6000 as carriers, the dissolution rate increased with increased amount of carriers. The best results among solid dispersions with Polyvinyl pyrrolidone K30 were obtained from the formulation A13 (Figure 3C). The increased dissolution rate may be due to the higher solubility of PVP K30 in dissolution medium and better wettability of Pioglitazone hydrochloride in the formulation. The regression coefficient (r) values for formulations A11 to B15 are tabulated in Table 5.

\subsubsection{Drug Release Kinetics Studies of Solid Dispersion}

The regression coefficient ' $r$ ' values were found to be higher in the Korsmeyer-peppas models, Hill equation model, Michaelis menten model and Weibull model respectively, indicating that the dissolution of pioglitazone from all formulations followed following above model.(Table 5).

\subsubsection{In-Vitro Drug Release Study of Solid Dispersion Incorporated Microcapsules}

The in vitro release profiles of nine formulations MC1 to MC9 are shown in Figure 8. It shows the plot of cumulative percent drug released as a function of time for different formulations. The cumulative percentage drug released indicates a controlled and prolonged drug release over an extended period of time. From the in vitro drug release profiles, it was observed that the drug release from microcapsules was decreased with an increase in cell forming material in the microcapsules (MC7, MC8, and MC9). The regression coefficient (r) values for formulations MC1 to MC9 are tabulated in Table 5.

\subsubsection{Drug Release Kinetics Studies of Solid Dispersion Incorporated Microcapsules}

The regression coefficient ' $r$ ' values were found to be higher in the zero order models, Hill equation model, Michaelis menten model, Korsmeyer peppas modal and Weibull model respectively, indicating that the dissolution of pioglitazone from all formulations followed following above model. The order of release rate observed with all microcapsules was MC8 >MC9>MC7>MC4>MC6>MC5 $>$ MC1 $>$ MC2 $>$ MC3. The drug release from the microcapsules was diffusion controlled.(Table 5).

\subsection{Muco-Adhesive Testing}

Microspheres with a coat consisting of alginate and a mucoadhesive polymer exhibited good muco-adhesive properties in the in vitro wash-off test. From the in vitro wash-off test it was observed that, the drug adherance the microcapsules from tissue was increase with an increase in muco-adhesive material in the microcapsules. The maximum adherence resulted in MC1, MC2 and MC3 
respectively and minimum found in MC9 and MC8. (Table 7).

\subsection{Analytical Testing}

\subsubsection{Fourier Transforms Infrared Spectroscopy}

FT-IR studies were done to detect the possible interactions between the Pioglitazone hydrochloride and carriers (polyvinyl pyrrolidone K30 and polyethylene glycol 6000). The characteristic peaks of Pioglitazone hydrochloride, polyvinyl pyrrolidone K30, polyethylene glycol 6000, physical mixtures and their formulations are given in Figure 4. Comparing the spectra of physical mixtures with those of solid dispersions prepared by using different methods revealed that there were no differences in the positions of the absorption bands, hence providing evidence for the absence of hydrogen bonding interactions in the solid state between Polyvinyl pyrrolidone K30 with Pioglitazone $\mathrm{HCl}$ under investigation. The absence of any significant change in the IR spectral pattern of drugpolymer physical mixture indicated the absence of any interaction between the drug and the polymer.

\subsubsection{Powder X-Ray Diffraction Study}

The diffraction spectra of Pioglitazone $\mathrm{HCl}$ and polyethylene glycol 6000 show numerous distinct peaks indicating that both are present in a highly crystalline state (Figure 5A, C) and the broad peaks of Polyvinyl pyrrolidone K30 indicating a amorphous state. The PXRD pattern of solid dispersion of sample A13 (Figure 5F) exhibits the characteristic broad peaks of polyvinyl pyrrolidone $\mathrm{K30}$ and crystalline pioglitazone $\mathrm{HCl}$, represent the amorphous state of sample A13. B13 represent the numerous distinct peaks indicating that sample B13 is present in a highly crystalline state (Figure $5 G)$.

\subsubsection{Compatibility Studies of Solid Dispersion Incorporated Microcapsules}

FTIR studies were done to detect the possible interactions between the drug and the polymers in the microcapsules. Figure 7 Show the IR spectrum's of drug and the polymers. Comparing the spectra of individual drug and polymers with those of microcapsules prepared by using different methods revealed that there were no differences in the positions of the absorption bands, hence providing evidence for the absence of hydrogen bonding interactions in the solid state between cell forming polymer (Sodium alginate) and Mucoadhesive polymer (Sodium alginate, Carbopol, Sodium CMC, HPMC) with pioglitazone $\mathrm{HCl}$ under investigation. The absence of any significant change in the IR spectral pattern of drugpolymer mixture indicated the absence of any interaction between the drug and the polymer (Figure 7).

\section{Conclusion}

The study clearly shows that addition of polyvinyl pyrrolidone $\mathrm{K30}$ to Pioglitazone $\mathrm{HCl}$ improved its dissolution rate. Mechanisms involved are solubilization and improved wetting of the drug in the polyvinyl pyrrolidone K30 rich micro-environment formed at the surface of drug crystals after dissolution rate compared with physical mixtures. No solid solution formation and no hydrogen bonding interaction between polyvinyl pyrrolidone K30 with pioglitazone $\mathrm{HCl}$ could be detected. The crystallinity of the drug was reduced in solid dispersion formulation with polymers i.e. polyvinyl pyrrolidone K30.

The study also indicated that the solid dispersion incorporated muco-adhesive microcapsules could be formulated by using cell forming polymer sodium alginate and muco-adhesive polymers (Carbopol, Sodium CMC and HPMC) by orifice ion gelation method. Increasing the concentration of cell forming polymer (sodium alginate) in microcapsule formulation decreases the rate of drug release dramatically and the mucoadhesive property increase with increasing the muco-adhesive polymer. The solid dispersion incorporated muco-adhesive microcapsules of all the formulated batches were spherical, discrete and free flowing. FTIR studies indicate that there were not any possible interactions between the solid dispersion (drug) and the polymers in the microcapsules. The drug content was found to be almost uniform in a batch of mucoadhesive microcapsules. Increasing the concentration of cell forming polymer (sodium alginate) in microcapsule formulation decreases the rate of drug release, best result were found in alginate Carbopol formulations in both cases.

\section{Reference}

[1] Sean C. Sweetman (Martindale), The complete drug reference, $33^{\text {rd }}$ edition, PHP London, 2008, p-333.

[2] Tripathi K.D., Essential of medical pharmacology, $5^{\text {th }}$ edition, Jaypee brothers medical publishers (p) ltd., New Delhi, 2004, pp.247-251.

[3] M. Shimano, Y. Tsuji, Y. Inden, K. Kitamura, Pioglitazone a peroxisome proliferator-activated receptor-gamma activator attenuates atrial fibrosis and atrial fibrillation promotion in rabbits with congestive heart failure, Hearth Rhythm, March 2008, 5, 3, 451-459.

[4] M. Sakagami, W. Kainoshita, K. Sakon, J. I. Sato, Y. Makino, Mucoadhesive beclomethasone microspheres for powder inhalation, there pharmacokinetic and pharmacodynamics evaluation, Journal of Controlled Release, 2002, 80, 207-218.

[5] J. Takinshima, H. Onishi, Y. Machida, Prolongd intestinal absorption of Cephradine with chitosan-coated ethyl cellulose microspheres in rats, Biopharm Bull, 2002, 25, 1498-1502.

[6] M. Cuna, M. J. Alonso, D. Torres, Preperation and in-vivo evaluation of mucoadhesive microspheres containing amoxicillinresin complexes for drug delivery to the gastric mucosa, Journal Pharm Biopharm, 2001, 51, 199-205.

[7] Khannan K., Manavalan R., Kelkar A.K., Defferential photometric estimation of chlorphenicol and benzocain, Indian Drug, 25 (3), 128-130.

[8] Lalhriatpuii T. C. and Kawathekar N., Derivative Specrtrophotometric estimation of Pioglitazone and Metformin $\mathrm{HCl}$, Indian drug, Nov. 2005, 42, 11.

[9] Higuchi T, Connoras KA. Phase-solubility techniques, ADV Anal Chem Instrum, 1965, 4, 117-210.

[10] K. P. R. Chowdary, Y. S. Rao, Design and in vitro and in vivo evaluation of mucoadhesive microcapsules of Glipizide for oral controlled release, AAPS pharm Sci Tech, 2003, 4(3), 87-92.

[11] P.C. Harry, T. Chandy, C. P. Sharma, Chitosan/calcium alginate microspheres for intestinal delivery of nitrofurantoin, Journal of Microencapsulation, 1996, 13, 319-329.

[12] K. P. R. Chowdary, L. Sriniwas, Mucoadhesive drug delivery system: A review of current status, Indian drugs, Sep 2000, 37(9), 400-406. 
[13] K.P.R. Chowdhary, V. Ratna, Preparation and evaluation of Cellulose acetate microcapsules of Diclofenac sodium for sustained release, Indian drug, 1992, 29(11), 4.

[14] H. Takahata, T. Osawa, M. Kobayashi, microencapsulation of benzoic acid derivatives using an enteric polymer by surface neutralization method and derivation of empirical equation for predicting film forming, Chem. Pharm. Bull., 1993, 41(6), 137143.

[15] Ahmed Mohammed G * B. P. Satish, G.B. K. Kiran , Formulation And Evaluation Of Gastric- Mucoadhesive Drug Delivery Systems Of Captopril, Journal of Current Pharmaceutical Research, 2010, 2(1), 26-32. 